\title{
The Electroweak Model Based on the Nonlinearly Realized Gauge Group
}

\author{
Andrea Quadri* \\ Physikalisches Institut, Albert-Ludwigs-Universität, Freiburg, Germany \\ \& Dipartimento di Fisica, Università di Milano \& INFN, Sezione di Milano, Italy \\ E-mail: andrea.quadri@mi.infn.it
}

\section{Daniele Bettinelli}

Physikalisches Institut, Albert-Ludwigs-Universität, Freiburg, Germany

E-mail: Daniele.Bettinelli@physik.uni-freiburg.de

\section{Ruggero Ferrari}

Center for Theoretical Physics, MIT, Cambridge Massachusetts 02139

\& Dipartimento di Fisica, Università di Milano \& INFN, Sezione di Milano, Italy

E-mail: ruggero.ferrari@mi.infn.it

\begin{abstract}
The electroweak model is formulated on the nonlinearly realized gauge group $\mathrm{SU}(2) \otimes \mathrm{U}(1)$. This implies that in perturbation theory no Higgs field is present. We discuss the effective action at the tree level, the Slavnov-Taylor identity (necessary for the proof of unitarity), the local functional equation (used for the control of the amplitudes involving the Goldstone bosons) and the subtraction procedure (nonstandard, since the theory is not power-counting renormalizable). Particular attention is devoted to the number of independent parameters relevant for the vector mesons; in fact there is the possibility of introducing two mass parameters. With this choice the relation between the ratio of the intermediate vector meson masses and the Weinberg angle depends on an extra free parameter. Uniqueness of the tree-level effective action of the nonlinearly realized electroweak model is established. The model is formulated in the Landau gauge for the sake of simplicity and conciseness.
\end{abstract}

6th International Workshop on Chiral Dynamics, CD09

July 6-10, 2009

Bern, Switzerland

\footnotetext{
* Postersession.
} 
The electroweak model can be formulated [1]-[3],[12] on the nonlinearly realized gauge group $S U_{L}(2) \otimes U_{Y}(1)$. We present here a consistent strategy for the all orders perturbative expansion in $\hbar$ which has been previously applied to the four-dimensional nonlinear sigma model [7]-[10],[13] and to the pure $S U$ (2) massive Yang-Mills theory [5],[6]. Since the representation of the gauge group is nonlinear, no Higgs field is present in the perturbative spectrum.

The presence of a local functional equation [13] (LFE) encoding the invariance of the Haar measure under local $S U_{L}(2)$ transformations establishes a hierarchy among 1-PI Green functions: the 1-PI amplitudes with at least one external pseudo-Goldstone leg (descendant amplitudes) are fixed in terms of those with no pseudo-Goldstone fields (ancestor amplitudes).

While there is an infinite number of divergent descedant amplitudes already at one loop level, only a finite number of divergent ancestor amplitudes shows up order by order in perturbation theory provided that the tree-level Feynman rules are compatible with the weak power-counting [10] (WPC) condition

$$
d(G) \leq(D-2) n_{L}+2-N_{B}-N_{F}
$$

where $n_{L}$ is the number of loops and $N_{B}, N_{F}$ the number of external gauge- and fermion-fields of the graph $G$. Thus in the limit $D=4$ the number of divergent one-particle-irreducible (1-PI) amplitudes is finite at fixed number of loops. Notice the unconventional UV degree of the fermion fields, a consequence of the presence of quadrilinear interactions involving two fermions and two pseudo-Goldstone in the nonlinearly realized gauge theory $[1,2]$.

The unique tree-level vertex functional compatible with the symmetries of the model and the WPC condition is $[1,2]$

$$
\begin{aligned}
& \Gamma^{(0)}=\Lambda^{(D-4)} \int d^{D} x\left(2 \operatorname{Tr}\left\{-\frac{1}{4} G_{\mu \nu} G^{\mu v}-\frac{1}{4} F_{\mu v} F^{\mu v}\right\}\right. \\
& +M^{2} \operatorname{Tr}\left\{\left(g A_{\mu}-\frac{g^{\prime}}{2} \Omega \tau_{3} B_{\mu} \Omega^{\dagger}-F_{\mu}\right)^{2}\right\}+M^{2} \frac{\kappa}{2}\left(\operatorname{Tr}\left\{\left(g A_{\mu}-\frac{g^{\prime}}{2} \Omega \tau_{3} B_{\mu} \Omega^{\dagger}-F_{\mu}\right) \tau_{3}\right\}\right)^{2} \\
& +\sum_{L}\left[\bar{L}\left(i \not \partial+g \not A+\frac{g^{\prime}}{2} Y_{L} \not B\right) L+\sum_{R} \bar{R}\left(i \not \partial+\frac{g^{\prime}}{2}\left(Y_{L}+\tau_{3}\right) \not B\right) R\right] \\
& \left.+\sum_{j}\left[m_{l_{j}} \bar{R}_{j}^{l} \frac{1-\tau_{3}}{2} \Omega^{\dagger} L_{j}^{l}-m_{q_{j}^{u}} \bar{R}_{j}^{q} \frac{1+\tau_{3}}{2} \Omega^{\dagger} L_{j}^{q}+m_{q_{k}^{d}} V_{k j}^{\dagger} \bar{R}_{k}^{q} \frac{1-\tau_{3}}{2} \Omega^{\dagger} L_{j}^{q}+h . c .\right]\right) .
\end{aligned}
$$

$L$ is an element of the set of the left fields (flavour eigenstates) of the three families. For the sake of notational convenience, in the above equation the right components are also formally gathered into doublets $R$ [1]. We use the $2 \times 2$ matrix notation for the $S U_{L}(2)$ gauge triplet $A_{\mu}$, the $U_{Y}(1)$ gauge field $B_{\mu}$ and the pseudo-Goldstone fields collected in the matrix $\Omega$

$$
\begin{aligned}
& A_{\mu}=A_{a \mu} \frac{\tau_{a}}{2}, \quad \Omega=\frac{1}{v}\left(\phi_{0}+i \tau_{a} \phi_{a}\right), \quad \Omega \in S U(2), \quad F_{\mu}=i \Omega \partial_{\mu} \Omega^{\dagger}=F_{a \mu} \frac{\tau_{a}}{2}, \\
& G_{\mu v}=\partial_{\mu} A_{v}-\partial_{v} A_{\mu}-i g\left[A_{\mu}, A_{v}\right], \quad F_{\mu v}=\partial_{\mu} B_{v}-\partial_{v} B_{\mu} .
\end{aligned}
$$

$F_{\mu}$ is the flat connection which enters into the Stückelberg mass terms in the tree-level vertex functional. The $S U_{L}(2)$ gauge transformations take the form

$$
\Omega^{\prime}=U \Omega \quad B_{\mu}^{\prime}=B_{\mu} \quad A_{\mu}^{\prime}=U A_{\mu} U^{\dagger}+\frac{i}{g} U \partial_{\mu} U^{\dagger} \quad F_{\mu}^{\prime}=U F_{\mu} U^{\dagger}+i U \partial_{\mu} U^{\dagger} \quad L^{\prime}=U L \quad R^{\prime}=R
$$


Under the $\exp \left(i \frac{\alpha}{2} Y\right) \in U(1)$ local transformations $\Omega$ is transformed on the right

$$
\begin{array}{ll}
e^{-i \frac{\alpha}{2} Y} \Omega e^{i \frac{\alpha}{2} Y}=\Omega V^{\dagger} & e^{-i \frac{\alpha}{2} Y} F_{\mu} e^{i \frac{\alpha}{2} Y}=F_{\mu}+i \Omega V^{\dagger} \partial_{\mu} V \Omega^{\dagger} \\
e^{-i \frac{\alpha}{2} Y} A_{\mu} e^{i \frac{\alpha}{2} Y}=A_{\mu} & e^{-i \frac{\alpha}{2} Y} B_{\mu} e^{i \frac{\alpha}{2} Y}=B_{\mu}+\frac{1}{g^{\prime}} \partial_{\mu} \alpha \\
e^{-i \frac{\alpha}{2} Y} L e^{i \frac{\alpha}{2} Y}=\exp \left(i \frac{\alpha}{2} Y_{L}\right) L & e^{-i \frac{\alpha}{2} Y} e^{i \frac{\alpha}{2} Y}=\exp \left(i \frac{\alpha}{2}\left(Y_{L}+\tau_{3}\right)\right) R,
\end{array}
$$

where $Y_{L}$ is the hypercharge of the fermionic left doublet $L$ and

$$
V(\alpha)=\exp \left(i \frac{\alpha}{2} \tau_{3}\right)
$$

Notice that due to the nonlinear constraint $\Omega^{\dagger} \Omega=1$ both symmetries are nonlinearly implemented. Gauge-fixing and the whole set of external sources required in order to implement the SlavnovTaylor (ST) identity and the LFE are omitted.

Remarkably the WPC plus the nonlinear symmetry forces the absence of anomalous interactions at tree-level. However, two independent mass terms for the gauge fields are allowed, one for the neutral and one for the charged vector meson. Consequently the ratio of the vector meson masses is not fixed anymore by the Weinberg angle. The additional parameter in the gauge boson mass sector is denoted by $\kappa$.

$\Lambda$ is an additional parameter which controls the energy scale of the radiative corrections. Since the model is non-renormalizable, a shift in $\Lambda$ cannot be compensated by a shift in the tree-level parameters.

Quantization is performed in the Landau gauge for the sake of simplicity and elegance. The extension to arbitrary 't Hooft $\alpha$-gauge can be carried out along the lines of Ref.[4]. The subtraction strategy must fulfill the following functional identities:

- the ST identity, necessary in order to guarantee Physical Unitarity order by order in the loop expansion [11];

- the LFE, encoding the hierarchy among the 1-PI Green functions;

- the Landau gauge equation.

Despite the fact that both the hypercharge and the $S U_{L}(2)$ symmetry are non linearly realized, the Ward identity for the electric charge has a linear form on the vertex functional.

Moreover, since the finite renormalizations associated with higher order invariants cannot be reinserted back into the tree-level vertex functional without spoiling either the symmetries or the WPC, we reject the interpretation of such finite renormalizations as additional bona fide physical parameters and we perform a minimal subtraction of properly normalized 1-PI ancestor amplitudes in order to fulfill all the above symmetry requirements.

This approach has been advocated in the context of the four-dimensional nonlinear sigma model in Refs. [8],[10],[13]. Non-trivial finite parts guaranteeing the fulfillment of the relevant functional identities at higher orders have been checked explicitly in Ref. [9].

In the proposed subtraction scheme the model is predictive. A first step towards a detailed study of the one-loop phenomenology of the nonlinear theory has been developed in Ref. [3] by evaluating the one-loop selfenergies of the gauge bosons. 
Physical unitarity cancellations have been checked and a detailed comparison of the self-energies with the SM case has been given.

We also provide a rough estimate both of the extra mass parameter and of the scale of the radiative corrections in a very simplified fit. We fix the parameters $g, g^{\prime}, M$ on measures taken at (almost) zero momentum transfer $\left(\alpha, G_{\mu}\right.$ and the $v-e$ scattering that provides a precise value for $\left.\sin ^{2} \theta_{W}\right)$, while the one-loop corrections are confronted with measures at the resonant value of the vector bosons energies. The resulting values are challenging: the departure from the Weinberg relation between the vector meson mass is very small and the scale of the radiative corrections is of the order of hundred $\mathrm{GeV}$ :

$$
\kappa=0.0085 \quad \Lambda=283 \mathrm{GeV}
$$

These values are quite encouraging for the calculation of further radiative corrections. However one should consider only the order of magnitude of these numbers. In fact they depend strongly from the value of $\sin ^{2} \theta_{W}$. Only a fit including other sensitive quantities will be able to reduce their variability.

If confirmed, the resulting scenario is then very interesting. For instance an Higgs boson could emerge as a non-perturbative mechanism, but then its physical parameters are not constrained by the radiative corrections of the low energy electroweak processes. Otherwise, our energy scale for the radiative corrections $\Lambda$ is a manifestation of some other high energy physics.

\section{References}

[1] D. Bettinelli, R. Ferrari and A. Quadri, Int. J. Mod. Phys. A 24 (2009) 2639 [arXiv:0807.3882 [hep-ph]].

[2] D. Bettinelli, R. Ferrari and A. Quadri, "The SU(2) X U(1) Electroweak Model based on the Nonlinearly Realized Gauge Group. II. Functional Equations and the Weak Power-Counting," arXiv:0809.1994 [hep-th].

[3] D. Bettinelli, R. Ferrari and A. Quadri, Phys. Rev. D 79 (2009) 125028 [arXiv:0903.0281 [hep-th]].

[4] D. Bettinelli, R. Ferrari and A. Quadri, J. General. Lie Theor. Appl. 2 (2008) 122 [arXiv:0712.1410 [hep-th]].

[5] D. Bettinelli, R. Ferrari and A. Quadri, Phys. Rev. D 77 (2008) 105012 [arXiv:0709.0644 [hep-th]].

[6] D. Bettinelli, R. Ferrari and A. Quadri, Phys. Rev. D 77 (2008) 045021 [arXiv:0705.2339 [hep-th]].

[7] D. Bettinelli, R. Ferrari and A. Quadri, JHEP 0703 (2007) 065 [arXiv:hep-th/0701212].

[8] D. Bettinelli, R. Ferrari and A. Quadri, Int. J. Mod. Phys. A 23 (2008) 211 [arXiv:hep-th/0701197].

[9] R. Ferrari and A. Quadri, JHEP 0601 (2006) 003 [arXiv:hep-th/0511032].

[10] R. Ferrari and A. Quadri, Int. J. Theor. Phys. 45 (2006) 2497 [arXiv:hep-th/0506220].

[11] R. Ferrari and A. Quadri, JHEP 0411 (2004) 019 [arXiv:hep-th/0408168].

[12] R. Ferrari, M. Picariello and A. Quadri, Phys. Lett. B 611 (2005) 215 [arXiv:hep-th/0409194].

[13] R. Ferrari, JHEP 0508 (2005) 048 [arXiv:hep-th/0504023]. 